\title{
Gestión documental en un grupo de investigación biomédica
}

\author{
Por Miguel Romero-Cuevas, Fernando Reviriego-Barquilla, María Marín-Martínez \\ y Fernando Rodríguez-De-Fonseca
}

\begin{abstract}
Resumen: Con el objeto de sistematizar los procesos y mejorar la calidad de las actividades de un grupo de investigación biomédica, se ha diseñado e implementado un sistema de gestión de la investigación, desarrollo e innovación $(I+D+i)$ basado en la norma UNE 166002:2006 (similar a la ISO 9001). Se describen las experiencias y resultados obtenidos durante la selección y adaptación de Open KM para la gestión de un grupo de investigación. Se ha demostrado la utilidad de este tipo de plataforma para este propósito, viéndose que podría ser exportada a otros grupos de investigación.

Palabras clave: Gestión $I+D+i$, Sistema de gestión documental, SGD, Investigación, Biomedicina, UNE 166002, Normas, Grupo de investigación.
\end{abstract}

Title: Document management system in a biomedical research group

Abstract: In order to systematize the processes and improve the quality of the activities of a biomedical research group, a research, development and innovation (RDI) management system based on the Spanish norm UNE 166002:2006 has been designed and implemented. We describe the experience and results obtained during the selection and adaptation of the OpenKM document management system platform as a useful means for research group management. The usefulness of this type of platform for this purpose is demonstrated, which could be exported to other research groups.

Keywords: RDI management, Document management system, DMS research, Biomedicine, UNE 166002, Research group, Standards.

Romero-Cuevas, Miguel; Reviriego-Barquilla, Fernando; Marín-Martínez, María; Rodríguez-De-Fonseca, Fernando. "Gestión documental en un grupo de investigación biomédica". El profesional de la información, 2010, mayo-junio, v. 19 , n. 3, pp. 300-307.

DOI: 10.3145/epi.2010.may.12

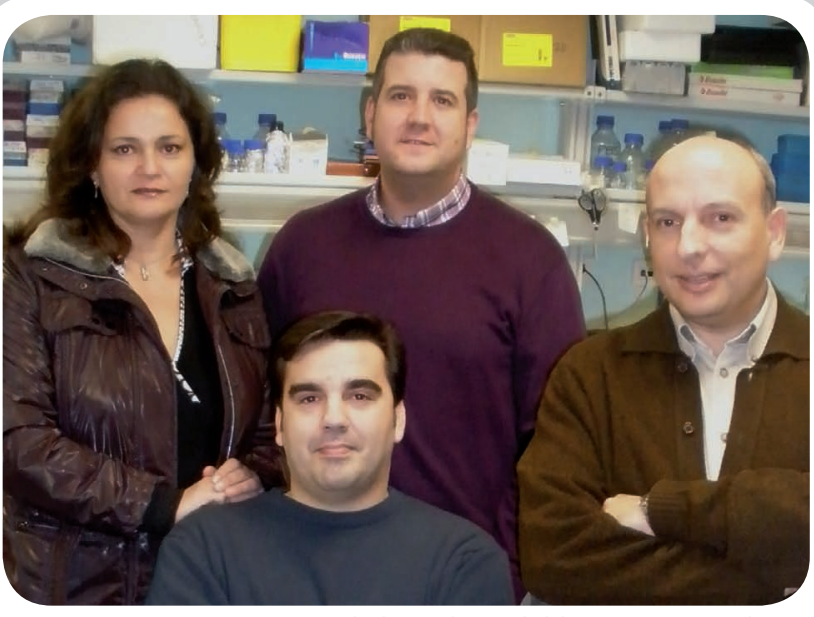

María Marín-Martínez es diplomada en biblioteconomía y licenciada en documentación por la Univ. de Granada. Trabaja como documentalista del Servicio de Documentación Científica de la Fundación Imabis en Málaga. Colabora en proyectos de edición electrónica y en actividades de teleformación mediante la plataforma Moodle.

Miguel Romero-Cuevas es doctor en biología y licenciado en bioquímica por la Univ. de Navarra. Desde el año 2004 ha desempeñado labores de gestión de la investigación biomédica, primero en la Oficina de Transferencia de la Fundación Imabis de Málaga y en la actualidad como gestor de proyectos e investigador del grupo de neuropsicofarmacología de la misma Fundación.

Fernando Rodríguez-De-Fonseca es doctor en medicina y cirugía por la Universidad Complutense de Madrid. Trabaja como asesor científico e investigador principal del grupo de neuropsicofarmacología de la Fundación Imabis.

Fernando Reviriego-Barquilla es ingeniero informático por la Universidad Politécnica de Madrid. Trabaja en la Fundación Imabis como programador de software biomédico y dando apoyo y asesoramiento tecnológico a los distintos grupos de investigación.

\section{Introducción}

EN LAS DOS ÚLTIMAS DÉCADAS la investigación biomédica en España ha experimentado un enorme impulso. Este crecimiento ha permitido mejorar los índices productivos de nuestra ciencia como generadora de conocimiento científico, compitiendo en igualdad de condiciones e incluso con ventaja con algunos países de nuestro entorno, especialmente en algunos campos de la investigación básica y clínica (Prieto-Carles; García-Romero, 2003).

Consecuencia de ello es la necesidad de crear y consolidar estructuras de gestión de la investigación, como las fundaciones, los centros de investigación biomédica en red (Ciber) y las redes temáticas de investigación cooperativa (Retics) del Instituto de Salud Carlos III, o los institutos de investigación biomédica. Asimismo se han im- pulsado medidas de formación y profesionalización de recursos humanos en el ámbito de la gestión de la investigación, como las ayudas predoctorales de formación en gestión de la investigación en salud (FGIN) dentro del actual Plan $\mathrm{Na}$ cional de $I+D+i$, en vigor para el período 2008-2011.

Sin embargo a pesar de los esfuerzos realizados, el aumento de los recursos de I+D+i se ha llevado a cabo a un ritmo menor que el cre- 
cimiento de la producción científica y las actividades de los grupos de investigación. Esta diferencia ha generado un déficit gestor, compensado por la asunción de tareas de planificación, organización y control por parte de los investigadores responsables de los proyectos que, si bien alcanzan la excelencia científica, pueden adolecer de falta de formación en metodología gestora. Así, las actividades de $\mathrm{I}+\mathrm{D}+\mathrm{i}$ se ven en ocasiones como procesos únicos, creativos y carentes de estructura, en los que sólo se tiene en cuenta la información científica de proyectos aislados, sin una integración dentro de una estrategia de evolución del conocimiento y su transferencia a la sociedad como progreso social y económico, que es el fin último de los proyectos de $\mathrm{I}+\mathrm{D}+\mathrm{i}$.

\section{"Una forma de tratar las carencias existentes en gestión es la optimización de los recursos disponibles mediante la aplicación de la norma UNE 166002:2006"}

\section{Estructura del grupo de investigación}

El grupo de investigación en neuropsicofarmacología pertenece a la fundación Instituto Mediterráneo para el Avance de la Biotecnología y la Investigación Sanitaria (Imabis), qué es una de las estructuras de gestión de la investigación biomédica de Andalucía. Su investigación se centra en el estudio de la anatomía, biología molecular, fisiología y farmacología de distintos sistemas fisiológicos implicados en la adicción, los trastornos del comportamiento, los trastornos alimenticios (obesidad y anorexia) y la diabetes.

Está compuesto por 20 personas: 10 doctores, 5 de los cuales son investigadores principales y uno es coordinador del grupo, y 10 licenciados, uno de los cuales se dedica a labores administrativas (tabla 1).

\begin{tabular}{|ll|}
\hline \multicolumn{1}{|c|}{ Personal } \\
\hline 1 & $\begin{array}{l}\text { doctor investigador principal } \\
\text { (coordinador) }\end{array}$ \\
\hline 4 & $\begin{array}{l}\text { doctores investigadores } \\
\text { principales }\end{array}$ \\
\hline 5 & doctores investigadores \\
\hline 9 & licenciados investigadores \\
\hline 1 & licenciado administrativo \\
\hline
\end{tabular}

Tabla 1. Plantilla del grupo de investigación en neuropsicofarmacología

Uno de los mayores problemas gestores del grupo es la dispersión de las localizaciones donde se realizan los distintos trabajos: dependiendo de distintas instituciones de investigación en la propia provincia de Málaga (Universidad de Málaga, Complejo Hospitalario Carlos Haya...), en el territorio nacional, con estancias en laboratorios de grupos colaboradores (Barcelona, Madrid, Sevilla...), y también estancias internacionales (Francia, Estados Unidos...).

Tras un análisis de la situación inicial de nuestro grupo en el que se midieron las variables de producción científica establecidas por organismos como el Instituto de Salud Carlos III en los procesos de evaluación de proyectos de investigación o en el curriculum vitae profesionalizado (Méndez-Vásquez et al., 2008), se constató el buen trabajo llevado a cabo en la realización de las tareas de investigación. Se observaron buenos resultados en la cantidad y la calidad de los indicadores de la actividad científica de los últimos 5 años (tabla 2), como publicaciones en revistas con factor de impacto del ISI Thomson Reuters, proyectos de investigación financiados por organismos públicos y privados, y aumento del número de citaciones.

Además de los indicadores de la actividad científica, se realizó un análisis de la gestión del grupo mediante entrevistas personales con los componentes y la recopilación dar el tratamiento de las carencias existentes en gestión es la optimización de los recursos disponibles mediante la aplicación de la norma UNE 166002:2006 de Gestión de la $I+D+i$ : requisitos del sistema de gestión de la $I+D+i$, elaborada por la Asociación Española de Normalización y Certificación (Aenor).

En este artículo se describe el diseño del sistema de gestión de I+D+i de un grupo de investigación biomédica basado en esta norma, y su puesta en funcionamiento mediante la utilización de un software de gestión documental.

\begin{tabular}{|l|r|r|r|r|r|r|}
\hline \multicolumn{1}{|c|}{ Indicador } & $\mathbf{2 0 0 5}$ & $\mathbf{2 0 0 6}$ & $\mathbf{2 0 0 7}$ & $\mathbf{2 0 0 8}$ & $\mathbf{2 0 0 9}$ & Total \\
\hline Publicaciones en revistas ISI & 13 & 12 & 18 & 30 & 23 & $\mathbf{9 6}$ \\
\hline Citas ISI (coordinador grupo) & 458 & 408 & 436 & 622 & 607 & $\mathbf{2 . 5 3 1}$ \\
\hline Congresos internacionales & 6 & 2 & 7 & 6 & 8 & $\mathbf{2 9}$ \\
\hline Congresos nacionales & 18 & 6 & 12 & 13 & 15 & $\mathbf{6 6}$ \\
\hline Tesis doctorales defendidas & 1 & 1 & 1 & 1 & 0 & $\mathbf{4}$ \\
\hline Capítulos en libros & 5 & 0 & 3 & 2 & 1 & $\mathbf{1 1}$ \\
\hline Solicitudes de patentes & 0 & 1 & 1 & 0 & 2 & $\mathbf{4}$ \\
\hline Proyectos con financiación pública & 6 & 3 & 4 & 5 & 2 & $\mathbf{2 0}$ \\
\hline Proyectos con financiación privada & 1 & 1 & 0 & 0 & 1 & $\mathbf{3}$ \\
\hline
\end{tabular}

Tabla 2. Resumen de la actividad investigadora del grupo en los últimos cinco años.

Fuente: Sistema de Información Científica de Andalucía (SICA) y Web of Science de ISI Thomson Reuters. 
de registros (curriculum vitae de los investigadores, documentos de calendarios de reuniones y seminarios, documentos de participación de investigadores en elaboración de propuestas, correos electrónicos de anuncio de convocatorias de actividades de formación, investigación...), cuyo análisis mostró debilidades en los siguientes aspectos:

- Falta de competencias en gestión de proyectos, con una deficiente estructura del grupo (piramidal) y una falta de eficiencia en la utilización de los recursos.

- Deficiente gestión de la información y el conocimiento con dificultad para la circulación libre del mismo. De modo rutinario se realizan distintas tareas aisladas dentro de proyectos sin tener una visión global e integradora de los resultados a obtener.

- Falta de aportación por parte de los investigadores no principales de ideas, iniciativas innovadoras y otras actuaciones para la captación de fondos.

- Falta de una cultura de difusión y transferencia entre los profesionales, y falta de proactividad en el apoyo y gestión para la protección de los resultados y la búsqueda de alianzas empresariales para la traslación de los mismos.

Estos resultados llevaron a la decisión de implementar un sistema de gestión y organización que pudiera corregir en la medida de lo posible las deficiencias detectadas.

\section{Diseño de un sistema de gestión basado en la Norma UNE: 166002 2006}

La primera medida adoptada para el diseño fue la profesionalización de la labor de gestión de la $\mathrm{I}+\mathrm{D}+\mathrm{i}$ dentro del grupo. Para ello se eligió al doctor que no era el coordinador del grupo que presentaba una mayor experiencia, además de un alto grado de conocimiento de la entidad y el mayor número de horas en acciones formativas en el campo de la gestión. De este modo, se le asignó el papel de gestor de proyectos con una dedicación del $80 \%$ a estas tareas (el 20\% restante investiga). En esta etapa, también se redirigió el trabajo de uno de los licenciados a prestar apoyo al gestor de proyectos, además de dar cobertura al proyecto en el que estaba asignado como administrativo.

En segundo lugar se llevó a cabo la reestructuración del organigrama del grupo de acuerdo con los requisitos de la Norma. Se creó la unidad de gestión de la I+D+i compuesta por todos los investigadores principales, el gestor de proyectos y el licenciado administrativo, como encargada de gestionar el sistema y los proyectos. Se definió la unidad de $\mathrm{I}+\mathrm{D}+\mathrm{i}$, compuesta por todos los investigadores (incluidos los principales), encargada de realizar las actividades de investigación propiamente dichas. Se consiguió de este modo una estructura organizativa más horizontal y participativa (figura 1).

\section{"Un SGD es una \\ herramienta adecuada para gestionar un grupo de I+D+i”}

Una vez reestructurado el organigrama se optó por un modelo de gestión por procesos. Este enfoque permite definir los indicadores con los que se evaluará el rendimiento de las distintas actividades, no sólo consideradas de forma aislada, sino formando parte de un conjunto estrechamente interrelacionado, que era una de las particularidades buscadas desde un principio (Beltrán et al., 2002).

La principal tarea fue el diseño y definición del mapa de procesos del grupo de investigación (figura 2). Según la Norma ISO 9000 un proceso es "un conjunto de actividades mutuamente relacionadas o que interactúan, las cuales transforman elementos de entrada en resul-

Unidad de Gestión de I+D+i
Compuesta por:
- Investigadores principales
- Gestor de proyectos
- Licenciado administrativo
Se encarga de:
- Implantación y mantenimiento del sistema de gestión
- Planificación y control de los indicadores internos de actividad del grupo
- Planificación y realización de la vigilancia tecnológica
- Propuesta y selección de ideas de proyectos innovadores
- Preparación de propuestas de investigación
- Planificación, realización y control de los proyectos de investigación
- Evaluación y protección de los resultados
- Transmisión y transferencia de los resultados
Compuesta por:
- Todos los investigadores
Se encarga de:
- Realización de vigilancia tecnológica
- Propuesta de ideas de proyectos innovadores
- Realización de las actividades de los proyectos de investigación
- Apoyo en la transmisión de los resultados

Figura 1. Componentes y funciones de la unidad de Gestión de la $I+D+i$ y de la unidad de $I+D+i$ 
tados". En el mapa de procesos del grupo se representan los procesos que componen el sistema, así como sus relaciones principales.

Los procesos se agrupan en tres grandes áreas relacionadas entre sí (figura 2):

\section{Procesos estratégicos}

Son aquellos cuya responsabilidad recae exclusivamente sobre la unidad de gestión de la I+D+i, y sobre todo marcarán la dirección a tomar por el grupo a medio-largo plazo:

a) Planificación estratégica: en la que se lleva a cabo la organización de las actividades a realizar.

b) Control: proceso realizado por el gestor de proyectos, que se encarga de tener la información de los indicadores internos del grupo en tiempo real: indicadores económicos, de actividades, de producción científica... En este sentido la obtención de los indicadores de producción bibliográfica es apoyada por personal experto perteneciente a la biblioteca de la fundación.

c) Alianzas: proceso a realizar por los investigadores responsables de proyectos para formar nuevas colaboraciones con grupos de investigación.

\section{Procesos de soporte}

Son proporcionados por el personal de gestión de la organización que acoge al grupo y da soporte a los procesos clave.

a) Recursos humanos: contratación y formación de los investigadores.

b) Gestión económica: recibe gestión de ingresos y facturación, gasto y contabilidad de los proyectos de investigación, así como apoyo en la petición y compra de material.

c) Infraestructuras: en las que se encuadran los espacios físicos y la maquinaria central con los que se realizan las actividades de $\mathrm{I}+\mathrm{D}+\mathrm{i}$.

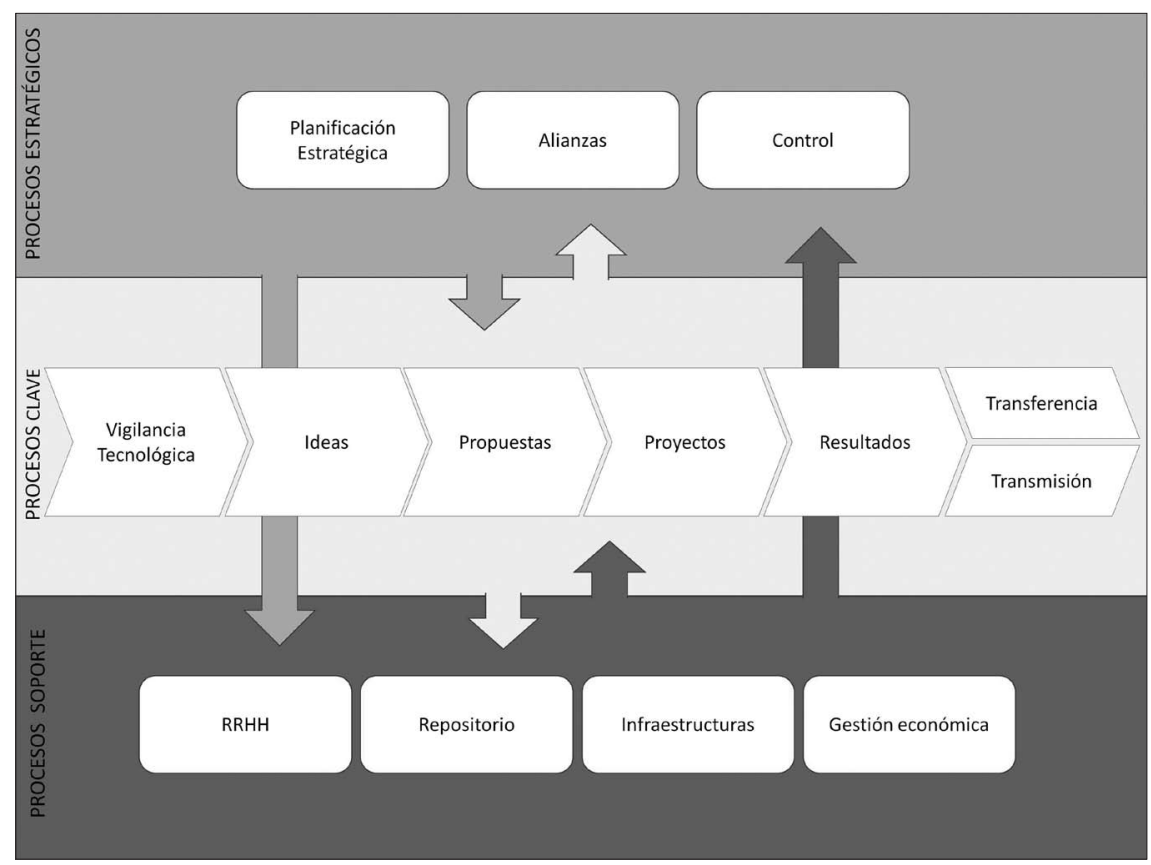

Figura 2. Mapa de procesos del grupo de investigación en neuropsicofarmacología.

d) Repositorio: en el que se encuentran los siguientes espacios de documentación:

- Biblioteca: donde se almacenan los documentos bibliográficos originales.

- Ayudas: espacio de ofertas y convocatorias de financiación de proyectos.

- Documentos oficiales: documentación administrativa oficial de la institución.

- Plantillas: donde están disponibles los documentos para realizar cualquier actividad: acta de reunión, informe de vigilancia tecnológica, propuesta de ideas...

\section{Procesos clave}

Son aquellos ligados directamente con la realización de las actividades de $\mathrm{I}+\mathrm{D}+\mathrm{i}$ encaminadas a lograr los objetivos: generación de conocimiento y transferencia y transmisión del mismo. Estos procesos son el eje central de la actividad del grupo:

a) Vigilancia tecnológica: consiste en la captura, el análisis, la difusión interna de la información necesaria y útil para el grupo de investigación, y su conversión en conocimiento (Muñoz-Durán; MarínMartínez; Vallejo-Triano, 2006). En este proceso cada miembro tiene asignado un tema de búsqueda, unas fuentes de información y una periodicidad (tabla 3 ).

b) Ideas: el proceso tiene como finalidad la selección de ideas originales presentadas por cualquier miembro para iniciar nuevas líneas y proyectos innovadores. Las propuestas recibidas son examinadas mensualmente por la unidad de gestión de la $\mathrm{I}+\mathrm{D}+\mathrm{i}$ para realizar la selección de las más factibles y que obtengan mejor puntuación según criterios estratégicos y operativos.

\section{"OpenKM se caracteriza por ser una aplicación sencilla, que ofrece mecanismos para la identificación, almacenamiento, seguimiento, recuperación y presentación de los documentos"}




\begin{tabular}{|l|l|l|l|}
\hline \multicolumn{1}{|c|}{ Personal } & \multicolumn{1}{|c|}{ Tema } & Periodicidad & \multicolumn{1}{c|}{ Fuentes de información } \\
\hline Investigador 1 & Patentes de fármacos antiobesidad & Bimestral & $\begin{array}{l}\text { Espacenet (Europa), Derwent Innovation } \\
\text { Index, USPTO (USA), OEPM (España) }\end{array}$ \\
\hline Investigador 2 & $\begin{array}{l}\text { Producción científica de potenciales } \\
\text { competidores }\end{array}$ & Mensual & Web of Science \\
\hline Investigador 3 & Nuevas tecnologías proteómicas en adicción & Trimestral & European Proteomics Association Bulletin \\
\hline Investigador 4 & Etc. & Etc. & Etc. \\
\hline
\end{tabular}

Tabla 3. Ejemplo de distribución de búsqueda y fuentes de información entre los miembros del grupo

c) Propuestas: se refiere a todas las actividades y la documentación asociada, memorias técnicas, presupuestos, certificados éticos para experimentación biomédica..., necesarias para formalizar una solicitud a un organismo público o privado para la financiación de tales propuestas.

d) Proyectos: conjuntos de actividades de $\mathrm{I}+\mathrm{D}+\mathrm{i}$ coordinadas $\mathrm{y}$ controladas con fechas de inicio y fin con el fin de lograr la generación de nuevo conocimiento o tecnología.

e) Resultados: son los datos de los experimentos realizados dentro de cada proyecto. Cada bloque de resultados es evaluado para decidir si es protegible mediante una patente, publicable en revistas científicas o en congresos, o si puede servir de base para acciones docentes. Por otro lado, si un resultado es susceptible de ser protegido, se iniciarán las tareas pertinentes antes de pasar a las de publicación. Como es sabido, la publicación de un invento invalida su patente, pues pierde su caracter de "novedad".

f) Transferencia: es el proceso mediante el cual los resultados de los proyectos son transferidos al tejido empresarial en tres formas principales:

- licencia de resultados protegidos,

- firma de un contrato de servicios de investigación o comercialización de productos, $\mathrm{y}$

- creación de una empresa spin-off a partir de una de las líneas de trabajo g) Transmisión: es la difusión del conocimiento generado en forma de publicaciones, acciones de divulgación, docencia y aportaciones a congresos.

\section{Implementación del sistema de gestión documental}

Una vez diseñado el sistema de gestión de la $\mathrm{I}+\mathrm{D}+\mathrm{i}$, se comenzó el trabajo para su puesta en funcionamiento. Para ello se decidió buscar un software orientado a la web, ya que una solución integral de intranet no podría solventar el problema de la deslocalización de los distintos componentes del grupo de investigación. Se estudiaron 4 tipos de plataformas web: gestores de proyectos y flujos de trabajo como dotProject; plataformas de formación, que se han descrito para la gestión del conocimiento en grupos de investigación (Uribe-Tirado; Melgar-Estrada; Bornacelly-Castro, 2007); redes sociales, en este caso se tenía experiencia de su aplicación en grupos de trabajo en el campo de la salud (Sánchez et al., 2008); y gestores documentales (Zhao et al., 2010).

\section{http://www.dotproject.net/}

Tras un análisis del manejo de información se comprobó que el principal elemento de trabajo eran los documentos, un recurso valioso donde quedan plasmadas las labores de investigación. La adopción de un criterio sistemático para la gestión de documentos resulta esencial para proteger y conservar los mismos como evidencia de todas las acciones realizadas (D'Alòs-Moner, 2006). Un sistema de gestión de documentos (SGD, o DMS por sus siglas en inglés) se consideró el mecanismo más adecuado para organizar el trabajo.

Se realizó un análisis detallado de tres SGDs de código abierto: Alfresco, Nuxeo y OpenKM, siendo esta última la opción elegida. OpenKM, además de adecuarse a nuestras necesidades funcionales, es una aplicación sencilla que ofrece mecanismos para la identificación, almacenamiento, seguimiento, recuperación y presentación de los documentos. Otra particularidad que nos llevó a su elección fue la usabilidad de su interfaz, más amigable y no tan difícil de usar como otras soluciones, debido a su similitud con el explorador de archivos de Windows.

http://www.alfresco.com/es/

http://www.nuxeo.com

http://www.openkm.com/

Tras la instalación del gestor documental se comenzó la elaboración del árbol de carpetas (figura 3) 


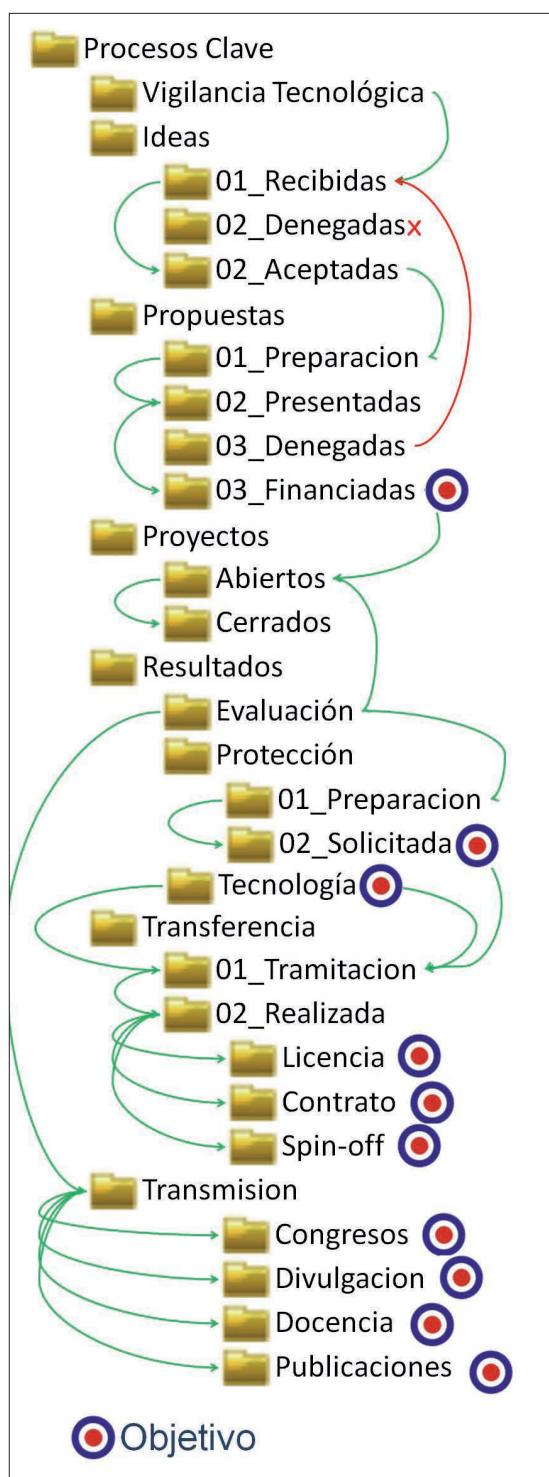

Figura 3. Árbol de carpetas de los procesos clave

basado en los procesos descritos en el diseño del sistema de gestión. Se crea una carpeta para cada bloque de procesos (estratégicos, clave, soporte), y dentro una carpeta por cada proceso. A continuación se describen los flujos de documentos generados en los procesos clave, que constituyen el conjunto de mayor importancia para la consecución de los objetivos del grupo.

a) Un informe de vigilancia tecnológica está formado por un documento plantilla con un texto explicativo de una extensión máxima de una cara de folio, que se sube a la carpeta correspondiente al proceso en el gestor documental.

b) Dentro del proceso ideas, hay 3 carpetas:
- 01_Recibidas: cualquier miembro del grupo puede subir un documento plantilla, que tiene una extensión máxima de una cara de folio, con 3 apartados: datos de contacto, resumen de la idea, posible fuente de financiación.

- 02_Denegadas: los documentos de las ideas denegadas se mueven a esta carpeta y pueden ser consultados por el autor de las mismas.

- 03_Aceptadas: con todas las ideas valoradas positivamente.

c) Las ideas aceptadas generan una nueva propuesta, que se encuentra en una de las carpetas siguientes según la fase del proceso en la que se esté:

- 01_Preparación: incluye la documentación técnica y administrativa para realizar la solicitud.

- 02_Presentadas: propuestas que ya han sido presentadas ante el organismo o empresa financiadora.

- 03_Denegadas: a ella se mueven las carpetas de propuestas que no han sido aprobadas por las entidades financiadoras. Deben entrar de nuevo en el flujo de trabajo como una idea nueva.

- 04_Financiadas: toda la documentación de las solicitudes de propuestas que han obtenido una valoración positiva por parte de un organismo financiador. Es de resaltar que en este punto ya se tiene un indicador de cumplimiento de objetivos de generación de actividad del grupo, que es el número de proyectos financiados.

d) Con cada propuesta financiada, se crea una entrada dentro en "proyectos abiertos", donde se encuentra toda la documentación de los proyectos en curso.

e) Resultados: están en documentos (hojas de cálculo, bases de datos, documentos de texto e hipertexto, multimedia, gráficos) y se encuentran en la carpeta de evaluación como fuente para preparar su publicación. También se hallan en la de protección o para realizar la solicitud de la misma. Además en la carpeta de tecnología están disponibles resultados cercanos a la transferencia (patentes concedidas, modelos de animales, técnicas...). Estas dos últimas carpetas constituyen información de cumplimiento de objetivos.

f) Transferencia: expedientes de licencias, contratos o spin-offs creados por el grupo.

g) Transmisión: publicaciones, acciones de divulgación, docencia y difusión en congresos realizadas por el grupo.

Por último, una vez realizado el árbol de carpetas, se crearon los roles de usuarios correspondientes y se asignaron permisos de acceso a las mismas.

\section{"Se observa una mayor implicación del personal en los procesos de búsqueda y obtención de recursos"}

\section{Resultados y conclusiones}

Después de 5 meses de utilización de la aplicación es pronto para evaluar el impacto sobre publicaciones, patentes y proyectos concedidos, pero la fase inicial (detección de oportunidades y presentación de propuestas) ha mejorado ostensiblemente. Un primer indicador de esta mejora detectado es la mayor implicación del personal en las actividades dentro de los procesos de búsqueda y obtención de recursos: vigilancia tecnológica, detección de oportunidades de financiación, generación de ideas y elaboración de propuestas, que han pasado de ser tarea casi exclusiva del coordinador del grupo a contar con la participación de los miembros de la unidad de gestión de $\mathrm{I}+\mathrm{D}+\mathrm{i}$. 


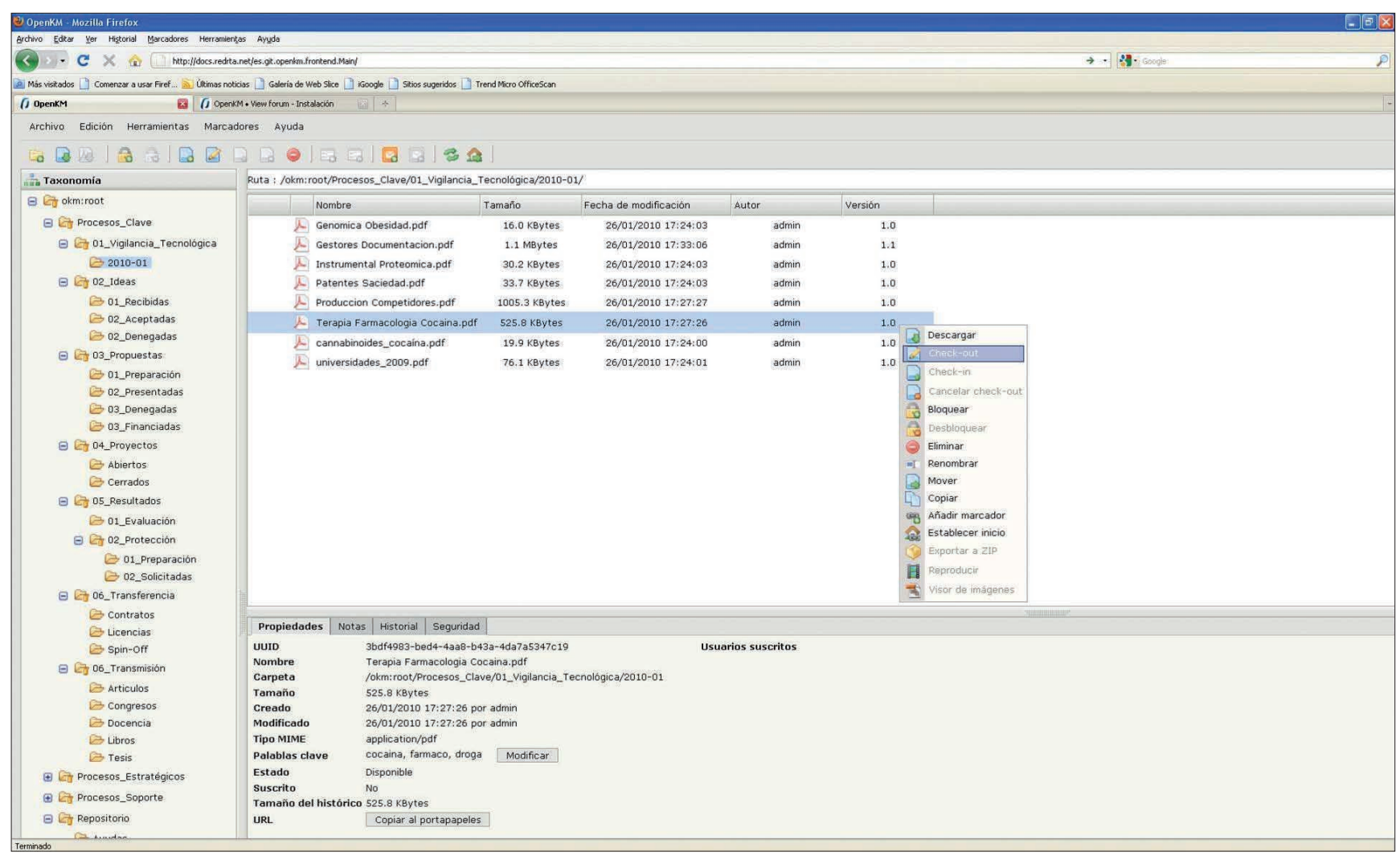

Figura 4. Espacio de trabajo en OpenKM

También en este bloque de actividades casi se ha duplicado el número de oportunidades de financiación detectadas y se ha multiplicado por 5 el número de propuestas de financiación solicitadas con respecto del mismo período del año anterior (tabla 4), además de diversificar las fuentes de financiación, con 3 solicitudes de proyectos o contratos europeos, 4 nacionales, 4 regionales y 1 proyecto privado. $\mathrm{La}$ calidad del aumento de actividad en estos procesos se podrá medir mediante el porcentaje de proyectos concedidos.

El sistema permite tener un espacio común donde recopilar todos los resultados, dando una visión más global de los mismos y permitiendo la actualización de los indicadores de resultados del grupo de investigación de un modo más fácil y rápido, con una simple visualización del árbol de carpetas y los documentos contenidos.

Por último es de resaltar la poca resistencia al uso del software por parte de los investigadores que rea- lizan el pilotaje debido a la semejanza con otros programas de uso cotidiano, y por funciones como el drag and drop de carpetas, el espacio de plantillas, control de versiones de documento, la opción de importar un path o conjunto completo de carpetas y subcarpetas desde un ordenador local (que permitió la rápida puesta en marcha de la plataforma), la posibilidad de definir cualquier carpeta como la de inicio (para ahorrar el tener que ir bajando en el árbol de carpetas desde el primer nivel), y un potente buscador basado en texto y marcadores de cada archivo.

Todos los resultados de las experiencias realizadas hasta el momento nos indican que un SGD es una herramienta adecuada para gestionar un grupo de $\mathrm{I}+\mathrm{D}+\mathrm{i}$. De hecho se han marcado dos objetivos próximos en esta línea de trabajo, que son exportar el sistema a un consorcio de grupos participantes en un proyecto a nivel europeo (Reprobesity), y a una red nacional de grupos de investigación (Red de Trastornos Adictivos).

http://www.reprobesity.eu http://www.redrta.es

\section{Bibliografía}

Beltrán-Sanz, Jaime; Carmona-Calvo, Miguel A.; Carrasco-Pérez, Remigio; Rivas-Zapata, Miguel A.; Tejedor-Panchon, Fernando. Guía para una gestión basada en procesos. Sevilla: Instituto Andaluz de Tecnología, 2002. ISBN 84923464-7-7.

http://www.iat.es/excelencia/html/subidas/ descarga/guiagestionprocesos.pdf

D’Alòs-Moner, Adela. "La gestión documental: aspectos previos a su implementación". El profesional de la información, 2006, mayo-junio, v. 15, n. 3, pp. 222-226. ISSN 1386-6710.

\begin{tabular}{|c|c|c|c|c|}
\hline Período & Personas & $\begin{array}{c}\text { Oportunidades } \\
\text { financiación }\end{array}$ & $\begin{array}{c}\text { Ideas } \\
\text { generadas }\end{array}$ & $\begin{array}{c}\text { Propuestas } \\
\text { elaboradas }\end{array}$ \\
\hline oct. 2008- feb. 2009 & 2 & 37 & 6 & 3 \\
\hline oct. 2009- feb. 2010 & 5 & 65 & 18 & 11 \\
\hline
\end{tabular}

Tabla 4. Comparación de indicadores de actividades de búsqueda y captación de recursos de $I+D+i$ antes y durante el pilotaje del SGD 
http://www.elprofesionaldelainformacion.com/ contenidos/2006/mayo/8.pdf

Méndez-Vásquez, Raúl-Isaac; Suñén-Pinyol, Eduard; Cervelló, Rosa; Camí, Jordi. "Mapa bibliométrico de España 1996-2004: biomedicina y ciencias de la salud". Medicina clínica, 2008, v. 130, n. 7, pp. 246-253. ISSN 0025-7753.

Muñoz-Durán, Javier; Marín-Martínez, María; Vallejo-Triano, José. "La vigilancia tecnológica en la gestión de proyectos de $\mathrm{I}+\mathrm{D}+\mathrm{i}$ : recursos y herramientas". El profesional de la información, 2006, noviembre-diciembre, v. 15 n. 6, pp. 411-419. ISSN 1386-6710.

http://www.elprofesionaldelainformacion.com/ contenidos/2006/noviembre/02.pdf

Norma 166002:2006. Gestión de la $I+D+i$ : requisitos de un sistema de gestión de $\mathrm{I}+D+i$. Madrid: Aenor, 2006

Prieto-Carles, Carlos; García-Romero, Antonio. "Evaluación de la investigación". En: Gutiérrez-Fuentes, José-Antonio; Puerta-López-Có- zar, José-Luis (ed.). Reflexiones sobre la ciencia en España. El caso particular de la biomedici na. Madrid: Fundación Lilly, 2003, p. 111-136. ISBN 978849567033.

Sánchez, Carlos L.; Romero-Cuevas, Miguel; López, Diego M.; Lorca, Julio; Alcázar, Francisco J.; Ruiz, Sergio; Mercado, Carmen Garcia-Fortea, Pedro. "Social network of Pesca (Open Source Platform for eHealth)". Studies in health technology and informatics, 2008, v. 137. pp. 340-345. ISSN 00926-9630.

Uribe-Tirado, Alejandro; Melgar-Estrada, Liliana-María; Bornacelly-Castro, Jaime-Alberto. "Utilización de Moodle en la gestión de información, documental y del conocimiento en grupos de investigación". El profesional de la información, 2007, septiembre-octubre, v. 16, n. 5, pp. 468-474. ISSN 1386-6710.

http://www.elprofesionaldelainformacion.com/ contenidos/2007/septiembre/09.pdf

Zhao, Wenle; Durkalski, Valerie; Pauls, Keith; Dillon, Catherine; Kim, Jaemyung; Kolk,
Deneil; Silbergleit, Robert; Stevenson, Valerie; Palesch, Yuko. "An electronic regulatory document management system for a clinical trial network". Contemporary clinical trials, 2010 enero, v. 31, n. 1, pp. 27-33

Miguel Romero-Cuevas, Fernando Reviriego-Barquilla, María Marín-Martínez, Fernando Rodríguez-De-Fonseca. Fundación Imabis, Málaga.

miguel.romero@fundacionimabis. org

fernando.reviriego@fundacionima bis.org

maria.marin@fundacionimabis.org fernando.rodriguez@fundacionima bis.org

\section{Revista científica y profesional en español líder en el área de información, bibliotecas y nuevas tecnologías de la información.}

El profesional de la información

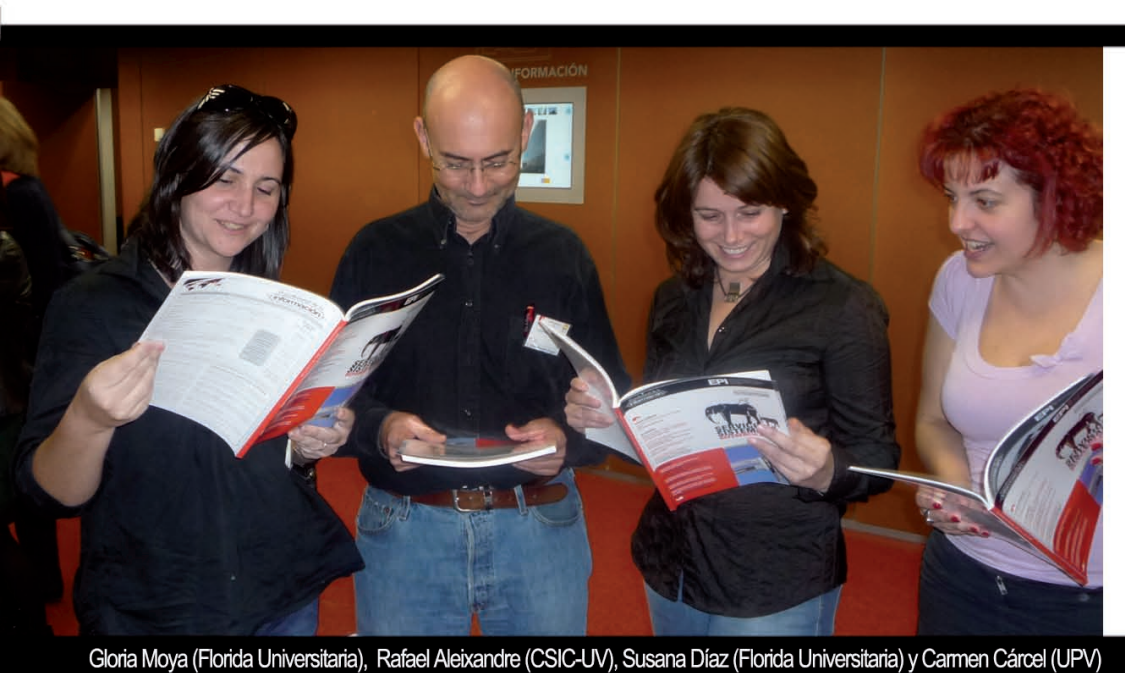

Gloria Moya (Florida Universitaria), Rafael Aleixandre (CSIC-UV), Susana Diaz (Florida Universitaria) y Carmen Cárœel (UPV

La 1a indexada por Science Citation Index

(Thomson ISI), y Scopus (Elsevier), además de otras 27 bases de datos y servicios bibliográficos.
Enero 2010 Sector editorial

Marzo 2010 Publicidad y comunicación empresarial

Mayo 2010 Información bio-médica

Julio 2010 Arquitectura de la información

Sept 2010 Cooperación de bibliotecas en red

Nov 2010 Medios de comunicación en intemet

Enero 2011 Psicología y sociología de la información

Marzo 2011 Archivos administrativos e intranets

Mayo 2011 Información de las administraciones públicas

\section{WwW.elprofesionaldelainformacion.com}

